\title{
Radar and Sonar Imaging and Processing (2nd Edition)
}

\author{
Andrzej Stateczny ${ }^{1, *(D)}$, Witold Kazimierski ${ }^{2}$ and Krzysztof Kulpa ${ }^{3}$ (D) \\ 1 Department of Geodesy, Gdansk University of Technology, 80-233 Gdansk, Poland \\ 2 Department of Geoinformatics, Maritime University of Szczecin, 70-500 Szczecin, Poland; \\ w.kazimierski@am.szczecin.pl \\ 3 Institute of Electronic Systems, Warsaw University of Technology, 00-665 Warszawa, Poland; \\ kkulpa@elka.pw.edu.pl \\ * Correspondence: andrzej.stateczny@pg.edu.pl; Tel.: +48-609-568-961
}

Citation: Stateczny, A.; Kazimierski, W.; Kulpa, K. Radar and Sonar Imaging and Processing (2nd Edition) Remote Sens. 2021, 13, 4656. https:// doi.org/10.3390/rs13224656

Received: 3 November 2021

Accepted: 16 November 2021

Published: 18 November 2021

Publisher's Note: MDPI stays neutral with regard to jurisdictional claims in published maps and institutional affiliations.

Copyright: (C) 2021 by the authors. Licensee MDPI, Basel, Switzerland. This article is an open access article distributed under the terms and conditions of the Creative Commons Attribution (CC BY) license (https:/ / creativecommons.org/licenses/by/ $4.0 /)$.

\begin{abstract}
The 14 papers (from 29 submitted) published in the Special Issue "Radar and Sonar Imaging Processing (2nd Edition)" highlight a variety of topics related to remote sensing with radar and sonar sensors. The sequence of articles included in the SI deal with a broad profile of aspects of the use of radar and sonar images in line with the latest scientific trends, in which the latest developments in science, including artificial intelligence, were used.
\end{abstract}

Keywords: radar; sonar; data fusion; sensor design; target tracking; target imaging; image understanding; target recognition

\section{Introduction}

Over the last few years, radar and sonar technology has been at the center of several major developments in remote sensing in both civilian and defense applications. Although radar technology has existed for more than 100 years, it is still developing and is now implemented in many maritime, air, satellite, and land applications. New technologies, including sparse image reconstruction and multistatic active and passive SAR and ISAR imaging, are changing the quality of images and areas of application. The rapid development of automotive radars in 3D dimensions, able to recognize different objects and assign the risk of collision, is one example of the progress of this technology. In maritime radars, aside from classical pulse radars, the application of FMCW technology is becoming increasingly popular. At the same time, sonar technology has also been used for dozens of decades, initially only for military solutions but, today, using 3D versions, it is being employed in many underwater tasks, such as underwater surface imaging, target detections, and tracking, among others. The impact of sonar technologies has been growing, particularly at the beginning of the autonomous vehicle era. Recently, the influence of artificial intelligence on radar and sonar image processing and understanding has emerged. Radar and sonar systems are mounted onboard smart and flexible platforms in addition to several types of unmanned vehicles. Both of these technologies focus on the remote detection of targets, and both may encounter numerous common scientific challenges. Unfortunately, specialists from the radar and sonar fields have barely interacted with each other, slowing down progress in both areas.

This Special Issue entitled "Radar and Sonar Imaging and Processing (2nd Edition)" is focused on the latest advances and trends in the field of remote sensing for radar and sonar image processing, addressing original developments, new applications, and practical solutions to open questions. Its aim is to increase data and knowledge exchange between the two communities and allow experts from other areas to understand the problems associated with radar and sonar.

Two major fields covered by the included papers are underwater acoustic image processing and synthetic aperture radar (SAR). In the case of SAR, various aspects of many systems were analyzed. Novel techniques and new algorithms are proposed for SAR 
signal processing, which are mostly focused on improving image quality by providing and implementing additional filtration. It is worth mentioning that all methods were thoroughly tested with real data that were gathered during online testing.

Topics covered in this Special Issue include:

- Passive and active radar imaging (SAR, ISAR);

- Passive, bistatic, and multistatic radar imaging;

- 3D radar and 3D sonar imaging;

- Sonar image processing, data reduction, feature extraction, and image understanding;

- Interferometric methods;

- Sparse image reconstruction;

- Automatic target detection and classification;

- Radar sensor design and platform developments;

- Radar and sonar target tracking and anti-collision algorithms and methods;

- Multisensor data fusion;

- Synergy between radar, sonar, and other sensors;

- Radar and sonar base autonomous navigation;

- Ground penetrating radar application in civil engineering;

- Automotive and maritime radar;

- Radar and sonar surveillance systems;

- Side scan sonar, imaging sonar, chirp sonar, and forward-looking sonar;

- Artificial intelligence for radar and sonar data processing.

In this article, a brief overview of the published papers and particularly the use of advanced modern technologies and data fusion techniques is provided. These two areas seem to represent the right direction for the future development of radar and sonar imaging and processing.

\section{Overview of Contributions}

Authors contributing to this Special Issue addressed many interesting subjects related to radar and sonar signal processing as generally understood. In fact, in the case of radar research, the focus was placed on various aspects of synthetic aperture radars (SARs), providing new approaches and methods to solve processing problems, filling some of the existing gaps, and providing solutions with improved accuracy and reliability. Different variants of SAR systems were investigated, and most of the research provided new algorithms verified with simulation and real data. Thus, a set of top-level papers was the result.

In $[1,2]$, the authors worked with signal processing in geosynchronous synthetic aperture radar (GEO SAR) imaging. Compared with low earth orbit SAR (LEO SAR), geosynchronous synthetic aperture radar (GEO SAR) has many advantages, such as its high resolution and wide swath and being unaffected by national boundaries and meteorological conditions. Recently, with the development of SAR systems, GEO SAR signal processing received increased attention, but higher resolution in SAR imaging also means more complex challenges. Despite being one of the most important sources of error in spaceborne SAR, elevation spatial variation is still not analyzed nearly enough in the literature. Therefore, the authors in [2] decided to focus on the analysis of this error. First, the decorrelation problems caused by spatial variation were presented. Second, through combination with SAR imaging geometry, the elevation spatial variation was decomposed into two-dimensional (2D) space variation of range and azimuth. Third, an imaging algorithm was proposed to solve the 3D space variation and improve the focusing depth. Finally, simulations with dot-matrix targets and distributed targets were performed to validate the imaging method. The analysis in that paper provides a theoretical basis for the future design of GEO SAR systems, and the improved imaging algorithm can be used to improve the focusing depth of scenes containing rough elevation information. The imaging algorithm proposed in that paper was used to carry out rough imaging of the slowly variant slope, and for the remaining part, the spatial variation error caused by fast variant part can compensated by the method of autofocus, which is the essence of the research presented in [1]. 
For a GEO SAR image with a complex scene, the elevation spatial variation error can be broken down into a slow variant slope and the remaining fast variant part. While the first was analyzed in [2], the second is the focus of [1]. Authors propose a block map-drift phase gradient autofocus (block-MD-PGA) algorithm to compensate for the random phase error part. Firstly, the source of the error is intuitively explained through the established geometric model. The authors then analyzed the error sufficiently to draw the conclusion that the error can be accurately fitted using a polynomial. Based on this conclusion, an autofocus compensation algorithm was proposed, and the simulations with dot-matrix targets and targets based on the TerraSAR-X image carried out. The results show that the proposed algorithm can compensate for the elevation variation error caused by the fast variant part well and can also allow adequately inversing the target elevation. The conclusion is also reached that the algorithm can work well under low-SNR conditions. The results obtained in $[1,2]$ are of great practical meaning and can be easily applied in GEO SAR wide swath terrain observation.

The subject of using scanning sonars for acoustic imaging of the seabed surface was addressed by Grzadziel in [3]. Such sonars are mainly used with tripods or special booms or are mounted onboard remotely operated or unmanned vehicles. Typical applications of scanning sonars include search and recovery operations, underwater infrastructure imaging, and fuzzy monitoring. The use of these sonars is often limited to shallow waters. Dive teams or underwater remotely operated vehicles (ROVs) are commonly used to survey shipwrecks, harbor piers, and ship hulls. However, limited underwater visibility, submerged debris, and extreme water depths can limit divers' capabilities. Grzadziel [3] propose a new nonstandard technique for using scanning sonar. The new application of the scanning sonar technique is a practical solution developed on the Polish Navy search and rescue ship "Lech". In order to verify the effectiveness of the proposed technique, the author took part in four different surveys conducted in the southeastern Baltic Sea. The surveys were performed using the MS 1000 scanning sonar. The results show that the proposed technique has the potential to provide detailed sonar images of the seabed and underwater objects before divers descend. The divers become familiar with the underwater situation, which undoubtedly increases the safety of the entire operation. Scanning sonar is unlikely to completely replace the work of divers, but it can reduce the number and duration of dives. The technique of using sonar proved useful during the rescue of the crew of a submarine that had settled to the bottom of the sea as part of a naval exercise. Sonar data obtained during four experimental trials conducted in the Baltic Sea demonstrate the validity, usefulness, and importance of the proposed technique, especially from the point of view of underwater work safety.

Another application of sonar in relation to [3] is the process of target tracking for providing information about objects in a specific area, which is one of the key issues affecting the safety of any vehicle moving in water. The main sensor used to track objects underwater is sonar, with one of the most popular configurations being forward-looking sonar (FLS). The state vector of the tracked target is typically estimated using numerical filter algorithms, such as the Kalman filter (KF) and its modifications, or the particle filter (PF). This requires the definition of a process model, including process noise, and a measurement model. Kazimierski and Zaniewicz [4] addressed the topic of tracking underwater moving objects in that they focused on aspects of process noise definition. This is usually implemented as Gaussian noise, with a covariance matrix defined by the author. Analytical and empirical analysis was conducted, including verification of existing approaches and a review of published literature. In addition, theoretical analysis of the factors affecting process noise was conducted, followed by empirical verification. The obtained results were subjected to discussion, which led to the formulation of conclusions. The results of the theoretical analysis were confirmed by an empirical experiment, and the obtained results were compared with commonly used process noise values in underwater target tracking processes. 
Another example of research focused on SAR image processing is given in [5]. This time, bistatic SAR systems were analyzed. Compared with the monostatic SAR system (MonoSAR), the BiSAR system could offer many additional advantages, such as frequent monitoring, reduced vulnerability, single-pass interferometry, capacity of forward- or backward-looking SAR imaging, and saved costs by using existing illuminators of opportunity with several receive-only systems. Generally, to obtain a good overlapping beam with airborne BiSAR, the transmitter and receiver antennas both operate in squint mode. However, the use of squint BiSAR echo brings more complexities for imaging due to its acquisition geometry. One of the challenges comes from the high-squint imaging algorithm, which is how to solve the large range cell migration (RCM), azimuth-dependent Doppler parameters, and the sensibility of the higher order terms when deriving the bistatic high-squint imaging algorithm. In that paper, a more accurate bistatic slant range approximation was introduced to apply the linear RCM correction (LRCMC) operation and improve the imaging accuracy of the algorithm. Combining the new slant range approximation and the idea of Lagrange inversion theorem (LIT) spectrum, a more accurate spectrum suitable for processing high-squint BiSAR data was introduced. Following this, a modified azimuth nonlinear chirp scaling (NLCS) operation was applied to eliminate the azimuth-dependence of Doppler parameters and equalize the azimuth frequency modulation for azimuth compression. The experimental results, with better focusing performance, demonstrate the high accuracy and effectiveness of the proposed algorithm.

Traditional manual horizon retrieval is time-consuming and labor-intensive, while automatic retrieval methods often suffer from their limited range of applications and discontinuity of retrieval results. In [6], the authors propose a new method for automatic horizon retrieval from sub-bottom profiles (SBPs) using an improved filtering algorithm. First, a clear and accurate image of the SBP was created using the intensity transformation method. Based on this, a new filtering method was proposed by improving the multiscale enhancement filtering algorithm to obtain clear horizons from the SBP image. The improvement was made by using a weighted vertical attenuation term based on the form of a logistic function, which was constructed using the eigenvectors of a Hessian matrix. The filtered image was then segmented using a thresholding method, and the horizon points in the SBP image were selected. Then, the horizon point merging method was applied, which uses the horizon directions to refine the selected horizon points. The proposed method was experimentally verified, obtaining satisfactory results.

Due to the influence of equipment and measurement environment instability, echo scattering, and other factors, it is sometimes difficult to obtain high-quality sub-bottom profile (SBP) images using traditional denoising methods. In [7], a novel SBP image denoising method is presented to obtain clean baseline images based on a nonlocal low rank structure. First, to exploit the layered structure of the SBP image, a direction image was obtained and used as a guidance image. Second, a robust guidance weight was given for accurate selection of similar patches. A novel denoising method combining weighting and nonlocal low rank filtering was proposed. Third, after discussing the filtering parameter settings, the proposed method was tested in real bottom measurements in both deep and shallow water. The experimental results confirm the excellent performance of the proposed method. Finally, the proposed method was verified and quantitatively compared with other methods based on synthetic images and achieved an overall peak mean signal-to-noise ratio (PSNR) of 21.77 and structural similarity index (SSIM) of 0.573, which is much better than other methods.

In radar measurements, one of the important issues is the radar cross-section (RCS) of the target. After processing RCS data, the scattering distribution and status information of targets can be obtained, such as radar SAR/ISAR imaging and radar automatic target recognition, etc. These can be estimated in the process of RCS reconstruction performed, for example, in anechoic chambers, using various techniques. In [8], the authors propose using a random frequency-hopping pattern to reduce the measurement time of the stepped frequency radar in anechoic chambers. In this pattern, the stepped frequency radar only randomly measures a part of the stepped frequencies in the bandwidth. The pattern is 
called the frequency-hopping pattern, and these measured RCS data are referred to as the frequency-hopping RCS sequence. Aiming at accurate RCS reconstruction, the authors proposed two variants of a nonparametric SPICE algorithm combined with the sparse GTD model (referred to as the GTD-SPICE method), which benefit from having global convergence properties. Numerical simulations on different parameters are separately carried out on each to evaluate their performance. The experimental results show that both the GTD-SPICE method and the DE-GTD-SPICE (damped exponential) method are reliable and effective in RCS reconstruction. In particular, the DE-GTD-SPICE method has a shorter computational time.

A tombolo is a narrow strip of land connecting the mainland with an island close to the shore and is formed by the depositing of sand and gravel by sea currents, most often resulting from natural phenomena. However, it can also be caused by human activity, as in the case of the pier in Sopot, a city located on the southern coast of the Baltic Sea in northern Poland $\left(\varphi=54^{\circ} 26^{\prime} \mathrm{N}, \lambda=018^{\circ} 33^{\prime} \mathrm{E}\right)$. As a result, the seabed is steadily rising, and the shoreline is shifting seaward. In addition, there is the additional disturbing phenomenon that the rising bottom sand is covering up the basin vegetation and threatening the resort character of the town. Removal of the sand to another location has been attempted several times. There is a lack of accurate geospatial data on the course of the tombolo seabed, its size, and its spatial pattern, caused only by bottom lowering in random places, and the progressive process of environmental degradation. In [9], the authors present the results of extensive and integrated geodetic and hydrographic measurements aimed at establishing a threedimensional model of the phenomena developing in Sopot. These measurements will allow the magnitude and rate of geospatial changes to be determined. Most modern geodetic and hydrographic methods were used in the study of these phenomena. The following methods were used to build the terrestrial part of the geospatial model: photos from photogrammetric flights (unmanned aerial vehicle (UAV)), laser scanning of the beach and the pier, and satellite orthophotos for the analysis of shoreline changes. For the marine component, bathymetric measurements were conducted using an unmanned surface vehicle (USV).

One of the interesting examples of SAR evolution is the linear array synthetic aperture radar (LASAR) presented in [10], which is one of the approaches for obtaining 3D SAR image. LASAR synthesizes the 2D equivalent array by moving the linear array; the 3D imaging results of the imaged scene are then obtained by combination with pulse compression technology. Compared with other approaches (such as TomoSAR and CurSAR), LASAR has a better antenna phase center (APC) control accuracy and a flexible moving trajectory. However, the realization of LASAR in real hardware systems is very complex and costly due to very high number of elements in linear array. Therefore, compressed sensing (CS) algorithms have been successfully introduced into LASAR imaging according to the sparsity of the LASAR imaging scene, as they can recover the original sparse signals by using random sampling signals. CS algorithms usually achieve high-quality sparse imaging at the expense of computational efficiency. To solve this problem, a fast Bayesian compressed sensing algorithm via relevance vector machine (FBCS-RVM) is proposed in [10]. The proposed method calculates the maximum marginal likelihood function under the framework of the RVM to obtain the optimal hyperparameters; the scattering units corresponding to the nonzero optimal hyperparameters are extracted as the target areas in the imaging scene. Then, based on the target areas, we simplify the measurement matrix and conduct sparse imaging. In addition, under low signal-to-noise ratio (SNR), low sampling rate, or high sparsity, the target areas cannot always be accurately extracted and probably contain several elements whose scattering coefficients are too small and closer to 0 compared to other elements. Those elements probably cause the diagonal matrix to be singular and irreversible and, thus, the scattering coefficients cannot be estimated correctly. To solve this problem, the inverse matrix of the singular matrix is replaced with the generalized inverse matrix obtained by the truncated singular value decomposition (TSVD) algorithm to correctly estimate the scattering coefficients. Based on the rank of the singular matrix, those elements with small scattering coefficients are extracted and 
eliminated to obtain more accurate target areas. Both simulation and experimental results illustrate that the FBCS-RVM algorithm successfully achieves sparse imaging with high quality and efficiency. It thus has improved computational efficiency and imaging quality compared to the other algorithms in the study and, therefore, the FBCS-RVM algorithm has great application prospects in 3D digital elevation models and urban area imaging.

Another approach to SAR imaging, presented in [11], is the multiple-input multipleoutput synthetic aperture radar (MIMO SAR) system. The MIMO concept is highly attractive for SAR systems, as a MIMO SAR system can obtain more equivalent channels than a traditional SAR system; this provides a promising method for solving the contradictions between high resolution, wide swath, and multidimensional challenges. The design of emitting waveforms is a difficult step in MIMO SAR systems. Various techniques have been proposed in this area, as presented in the paper. The demands for miniaturization and high gain of antenna are increasing. The digital array-fed reflector antenna has virtues such that it can play an important role in such a system. However, the geometric and signal models based on a reflector antenna are considerably different, with greater complexity and difficulties arising from the directly radiating planar antenna. As a result, the applications of the reflector antenna in the SAR system are not as mature as those of the planar antenna. A combination of multidimensional waveform encoding (MWE) technique and digital beamforming $(\mathrm{DBF})$ technology at the receiving end can greatly improve the MIMO SAR system performance, especially in terms of ambiguity suppression and waveform separation. Thus, the study presented in [11] combines digital array-fed reflector antenna and the DBF technique in the elevation direction for MWE SAR waveform separation. A DBF method, which is processed in the range-Doppler domain, is proposed. The method enables waveform separation without ambiguity at the receiver. Then, conventional SAR imaging methods are enabled, and an ideal SAR image is acquired. The simulation results show the correctness and feasibility of this method, which can obtain the ideal image without range ambiguity. The simulation for full polarization SAR can also show the great potential of the proposed method in applications of multidimensional radar observation. There are some limitations, which can be avoided in future works using various means and, therefore, the presented method has great potential for implementation.

Inverse synthetic aperture radar (ISAR) is a valuable modification of the SAR concept. It plays a crucial role in the detection, recognition, or identification of rapidly spinning targets because electromagnetic images of noncooperative targets with rapidly rotating motions can be obtained. The algorithm for this is proposed in [12]. Conventional studies of ISAR imaging have mainly focused on monostatic radar configurations in which a transmitter and a receiver are collocated, though in research, a bistatic approach is increasingly being undertaken. Compared with monostatic ISAR, the configuration for bistatic radar, in which the transmitter and receiver are spatially separated, presents advantages in radar detection scope, concealment, anti-interference capability, and the SNR of the received echo. Signal processing is, however, more complex, and various aspects and corrections have to be considered. One of these is the range of cell migration (RCM) correction. To consider the large RCM and nonstationary DFM of targets in the case of rapidly spinning motion, motived by the property of azimuth spatial invariance, a fast bistatic ISAR imaging method to target rapid spinning motions via exploiting the SAR technique was proposed in that work. In the case of the rapidly spinning targets in bistatic ISAR, the large RCM and nonstationary DFM of echo signals in coherent processing intervals restrain well-focused bistatic ISAR imaging, which creates difficulties in the recognition of targets. To overcome those obstacles, in [12], a high-efficiency bistatic ISAR imaging approach with circular shift operation in the RD domain is proposed based on the azimuth spatial invariance. To that end, first, the echoes of rapidly spinning targets are transformed into the RD domain, and their accurate analytical derivation is simultaneously derived based on the principle of stationary phase (POSP). Second, by utilizing an efficient circular shifting operation in the range-Doppler domain, the large RCM is corrected, and the time-varying DFM can also be compensated along the rotating radius direction. Finally, well-focused bistatic ISAR images 
are generated. Compared with existing methods, the proposed method has advantages in terms of its computational complexity, avoiding interpolation and multidimensional search operation, and in its satisfactory imaging performance under low signal-to-noise ratio (SNR) conditions thanks to the utilization of two-dimensional coherent integration gain. Finally, several numerical simulations are conducted to demonstrate the validity of the proposed algorithm. In conclusion, the proposed approach provides a good tradeoff between the performance and computation time in obtaining clear images for noncooperative targets with rapid spinning motions in a noisy environment.

Another vital technique for modern remote sensing is a multichannel high-resolution and wide-swath (HRWS) synthetic aperture radar (SAR). For the traditional single-channel SAR system, a low pulse repetition frequency (PRF) is required to obtain a wide swath image, which is in contrast with the demand of high PRF that is required in azimuth to avoid ambiguity. To solve this contradiction, in the multichannel SAR technique, one of the channels transmits the signals with low PRF to ensure a wide swath, and all channels that are set up along the track simultaneously receive signals to increase the PRF in the multichannel system. However, this solution suffers from various errors, among which the most important are amplitude error, phase error, and baseline error. Taking them into consideration, a novel sub-image local area minimum entropy reconstruction method (SILAMER) is proposed in [13]. The proposed method consists of three steps: sub-image generation, reconstruction coefficient and compensation phase estimation, and unambiguous reconstruction. First, according to the idea of image domain reconstruction, the sub-images are generated by employing the back-projection algorithm. During the sub-image imaging, the nonlinear motion of the SAR platform can be accurately compensated due to coherent accumulation. Then, the authors proposed an algorithm to estimate the reconstruction coefficient and the compensation phase, where a local area of the sub-image was used as the estimation input to improve the efficiency. For the estimation algorithm, as the closed form solution based on minimum image entropy criteria cannot be obtained, the conjugate gradient method was used to solve the optimization problem, which minimizes the entropy of the reconstructed image through iteration. Finally, the sub-images were weighted by the estimated reconstruction coefficient and corrected according to the compensation phase to obtain an unambiguous reconstructed image. The experimental results with real data verified the effectiveness of the proposed method, which can simultaneously correct amplitude error, baseline error, and phase error by using the estimated reconstruction coefficient and the compensation phase. Noticeably, the proposed algorithm has two additional advantages, i.e., (1) it can perform well under the condition of low signal-to-noise ratio (SNR), and (2) it is suitable for curved trajectory SAR reconstruction. The simulations also verify the advantages of the proposed method, confirming the usefulness of the approach.

Acoustic backscattering data are widely used to study the characteristics of seafloor sediment distribution. However, streaking and mosaic errors in the backscattered images lead to the introduction of interfering information into the feature extraction process, which is performed by a convolutional neural network or autoencoder. Moreover, the performance of existing classifiers is limited by such incorrect information, which means that it is difficult to achieve accurate classification in the study areas. In [14], a sediment classification method based on acoustic backscattering image is proposed by combining a denoising autoencoder (SDAE) and a modified extreme learning machine (MELM). SDAE is used to extract deep-rooted sediment features so that the training network can automatically learn to remove residual errors from the original image. The MELM model, which integrates weighted estimation, Parzen window, and particle swarm optimization, is used to attenuate the interference of mislabeled samples on the training network and to optimize the random expression of input layer parameters. Experimental results show that the SDAE-MELM method significantly reduces the mutual interference between sediment types, and the sediment boundaries are clear and continuous. The reliability and robustness of the proposed method are better than those of other approaches and were evaluated through the overall classification effect and comprehensive indices. 


\section{Conclusions}

The Special Issue entitled "Radar and Sonar Imaging Processing (2nd Edition)" comprises 14 articles on many topics related to remote sensing with radar and sonar sensors. In this editorial, short introductions of the published articles were presented.

It should be emphasized that radar and sonar image data processing is still one of the leading scientific topics, and much work is being undertaken in this area worldwide. New techniques and methods for extracting information from radar and sonar sensors and data have been proposed and verified. Some of them will prompt further research, while others are already mature and can be directly considered for industrial implementation and development.

Author Contributions: A.S. wrote the first draft, A.S. revised and rewrote the radar section, W.K. revised and rewrote the sonar section, K.K. read the final version. All authors have read and agreed to the published version of the manuscript.

Funding: This research received no external funding.

Institutional Review Board Statement: Not applicable.

Informed Consent Statement: Not applicable.

Data Availability Statement: Not applicable.

Acknowledgments: We would like to thank all the authors who contributed to the Special Issue and the staff in the editorial office.

Conflicts of Interest: The authors declare no conflict of interest.

\section{References}

1. Chang, F.; Li, D.; Dong, Z.; Huang, Y.; He, Z. Elevation Spatial Variation Error Compensation in Complex Scene and Elevation Inversion by Autofocus Method in GEO SAR. Remote. Sens. 2021, 13, 2916. [CrossRef]

2. Chang, F.; Li, D.; Dong, Z.; Huang, Y.; He, Z.; Chen, X. Elevation Spatial Variation Analysis and Compensation in GEO SAR Imaging. Remote. Sens. 2021, 13, 1888. [CrossRef]

3. Grzadziel, A. Results from Developments in the Use of a Scanning Sonar to Support Diving Operations from a Rescue Ship. Remote. Sens. 2020, 12, 693. [CrossRef]

4. Kazimierski, W.; Zaniewicz, G. Determination of Process Noise for Underwater Target Tracking with Forward Looking Sonar. Remote. Sens. 2021, 13, 1014. [CrossRef]

5. Li, C.; Zhang, H.; Deng, Y. Focus Improvement of Airborne High-Squint Bistatic SAR Data Using Modified Azimuth NLCS Algorithm Based on Lagrange Inversion Theorem. Remote. Sens. 2021, 13, 1916. [CrossRef]

6. Li, S.; Zhao, J.; Zhang, H.; Bi, Z.; Qu, S. A Novel Horizon Picking Method on Sub-Bottom Profiler Sonar Images. Remote. Sens. 2020, 12, 3322. [CrossRef]

7. Li, S.; Zhao, J.; Zhang, H.; Bi, Z.; Qu, S. A Non-Local Low-Rank Algorithm for Sub-Bottom Profile Sonar Image Denoising. Remote. Sens. 2020, 12, 2336. [CrossRef]

8. Li, Y.; Zhang, W.; Tian, B.; Lin, W.; Liu, Y. Scattering Model-Based Frequency-Hopping RCS Reconstruction Using SPICE Methods. Remote. Sens. 2021, 13, 3689. [CrossRef]

9. Specht, M.; Specht, C.; Mindykowski, J.; Dąbrowski, P.; Maśnicki, R.; Makar, A. Geospatial Modeling of the Tombolo Phenomenon in Sopot using Integrated Geodetic and Hydrographic Measurement Methods. Remote. Sens. 2020, 12, 737. [CrossRef]

10. Tian, B.; Zhang, X.; Li, L.; Pu, L.; Pu, L.; Shi, J.; Wei, S. Fast Bayesian Compressed Sensing Algorithm via Relevance Vector Machine for LASAR 3D Imaging. Remote. Sens. 2021, 13, 1751. [CrossRef]

11. Wang, S.; Sun, Y.; He, F.; Sun, Z.; Li, P.; Dong, Z. DBF Processing in Range-Doppler Domain for MWE SAR Waveform Separation Based on Digital Array-Fed Reflector Antenna. Remote. Sens. 2020, 12, 3161. [CrossRef]

12. Yang, Z.; Li, D.; Tan, X.; Liu, H.; Liao, G. A Fast Bistatic ISAR Imaging Approach for Rapidly Spinning Targets via Exploiting SAR Technique. Remote. Sens. 2020, 12, 2077. [CrossRef]

13. Zhou, L.; Zhang, X.; Zhan, X.; Pu, L.; Zhang, T.; Shi, J.; Wei, S. A Novel Sub-Image Local Area Minimum Entropy Reconstruction Method for HRWS SAR Adaptive Unambiguous Imaging. Remote. Sens. 2021, 13, 3115. [CrossRef]

14. Zhou, P.; Chen, G.; Wang, M.; Chen, J.; Li, Y. Sediment Classification of Acoustic Backscatter Image Based on Stacked Denoising Autoencoder and Modified Extreme Learning Machine. Remote. Sens. 2020, 12, 3762. [CrossRef] 